\title{
AJUSTE DE UM MODELO DE ARBITRAGEM DE PREÇOS SOB INCERTEZAS INFLACIONÁRIAS
}

\author{
M. Eduarda Tannuri* \\ Geraldo S. Souza* \\ Alain Caron*
}

\section{Resumo}

Investiga-se a adequabilidade do modelo de arbitragem de preços sob incertezas inflacionárias para o mercado brasileiro. Isto é feito através do ajuste estatístico deste modelo a série histórica dos retornos nominais dos ativos de maior liquidez na Bolsa de Valores de São Paulo. As hipóteses mantidas no modelo de arbitragem são validadas via a análise fatorial dos retornos dos ativos pelo método de extração dos fatores principais.

\begin{abstract}
This article investigates the adequability of an arbitrage princing theory (APT) model under uncertain inflation to the Brazilian stock market. It is carried out through the statistical adjustment of this model to the historical series of the nominal asset returns of the more traded stocks in the So Paulo stock exchange. The hypotheses are validated by the factorial analysis of the asset returns adopting the principal factors method.
\end{abstract}

\section{Introdução.}

A Teoria de Arbitragem de Preços (TAP), desenvolvida originalmente por Ross (1976), Ross (1977) e Roll e Ross (1980), é a infraestrutura teórica da qual se obtém os modelos de equilíbrio parcial que explicam o retorno de ativos financeiros em ambientes estáticos e dinâmicos. Neste artigo faremos uso do modelo tipo TAP de Elton, Gruber e Rentzler (1983) - EGR, que se propõe a explicar o comportamento do retorno de ativos financeiros sob incertezas inflacio nárias. Nossa aplicação contempla o mercado acionário de São Paulo no período de 1976-1985 e tem por objetivo o estudo da adequabilidade do EGR as condições do mercado acionário brasileiro (teste empírico).

*Universidade de Brasilia

R. de 2 conometria Rio de Janeiro v. 13, no 2, p.167-179 novembro 1993/abril 1994 
O modelo EGR difere da estrutura clássica de um modelo TAP na medida em que admite, a priori, que

retornos dos ativos é linear em dois fatores. Os fatores deste contexto são variáveis econômicas observáveis - $a$ inflação e um índice de mercado. O TAP, vale mencionar, postula uma estrutura em construtos abstratos independentes, i.e., um modelo clássico de análise fatorial. Tais construtos não tem significado econômico direto.

Faz-se mister observar aqui que a formulação do modelo EGR, em termos nominais, é equivalente a do modelo CAPM (Capital Asset Pricing Model) intertemporal de Merton (1973). Contudo as hipóteses utilizadas no modelo EGR são bem mais fracas. As restrições típicas do CAPM intertemporal são normalidade dos retornos e função de utilidade para os investidores na família Von NeumannMorgenstern.

O ambiente do modelo EGR é o de uma economia que oferece $n$ ativos de mercado (risco) e um ativo sem risco $F$. O resultado básico que permite o ajuste do modelo EGR a observações empíricas é uma conseqüência do Teorema da Separação em Três Fundos de Merton (1973). Quando o fator inflação está efetivamente associado a um prêmio de risco, i.e., recebe um preço, este teorema mostra que o retorno de um ativo fica completamente caracterizado por uma combinação linear dos retornos de $F$, de um portfólio de mercado $M$ e de um outro portfólio de ativos cujos retornos não se correlacionam aos fatores de mercado e a inflação.

Nossa exposição prossegue do modo seguinte. Na Seção 2 apresentamos de modo sucinto o modelo EGR. Na Seção 3 descrevemos as medidas utilizadas e os resultados de nosso exercício econométrico. Finalmente, na Seção 4, apresentamos um resumo das conclusões obtidas.

\section{A especificação EGR.}

O objetivo do modelo EGR é o de obter uma representação TAP dependente de fatores econômicos diretamente observáveis. Especificamente, dependente do nível de inflação e de um índice intimamente relacionado aos ativos financeiros presentes no mercado. Inicialmente o modelo é formulado em termos reais. Deste modo seja $r_{i}$ o retorno 
real do ativo $i$. Seja $r_{m}^{\prime}$ o retorno real de um portfólio que envolve todos os ativos disponíveis na economia em proporções estritamente positivas (inclusive do ativo $F$ ). Vê-se, assim, que $r_{m}^{\prime}$ funciona como um índice de mercado. Seja $\pi$ a taxa de inflação. No modelo EGR postula-se que

$$
r_{i}=\beta_{0}+\beta_{1 i} r_{m}^{\prime}+\beta_{2 i} \pi+\epsilon_{i}
$$

onde as variáveis $\epsilon_{i}$ representam componentes de erros estocásticos, homoscedásticos e que variam independentemente de ativo para ativo.

É importante observar que os fatores comuns a todos os ativos, i.e., o índice de mercado e a inflação, captam as componentes sistemáticas de risco. A componente estocástica reflete a interação de vários fatores de risco que influenciam no retorno dos ativos mas que de per si são de magnitude desprezável relativamente a parte sistemática do modelo.

Suponha, como o fazem EGR, a existência de um portfólio de arbitragem, contendo $l$ ativos, em proporções $x_{i}$, que satisfaça as hipóteses seguintes:

1. O portfólio tem investimento líquido nulo, i.e., tem-se

$$
\sum_{i=1}^{l} x_{i}=0 .
$$

Note que, por convenção, uma proporção é negativa quando se refere a venda a descoberto de um ativo.

2. O portfólio tem risco sistemático nulo em ambos os fatores, i.e.,

$$
\sum_{i=1}^{l} x_{i} \beta_{1 i}=0
$$

e

$$
\sum_{i=1}^{l} x_{i} \beta_{2 i}=0 .
$$

3. O portfólio é diversificado (tem risco não sistemático nulo), i.e.,

$$
\sum_{i=1}^{l} x_{i} \epsilon_{i}=0 \text {. }
$$


Segue das condições 1, 2 e 3 que o retorno esperado do portfólio de arbitragem é nulo, i.e.,

$$
\sum_{i=1}^{l} x_{i} r_{i}=0
$$

Pode-se então escrever,

$$
E\left(r_{i}\right)=a_{0}+a_{1} \beta_{1 i}+a_{2} \beta_{2 i}
$$

i.e., o vetor de retornos reais esperados pode ser escrito como uma combinação linear de um vetor unitário e dos vetores formados pelos coeficientes de sensibilidade aos fatores de risco sistemático. A vantagem desta representação é que permitirá o teste empírico do modelo EGR, como veremos adiante. Neste contexto, tem papel importante, a seguinte caracterização dos coeficientes de sensibilidade:

$$
\begin{aligned}
& \beta_{1 i}=\frac{\operatorname{cov}\left(r_{i}, r_{m}^{\prime}\right) \operatorname{var}(\pi)-\operatorname{cov}\left(r_{m}^{\prime}, \pi\right) \operatorname{cov}\left(r_{i}, \pi\right)}{\operatorname{var}\left(r_{m}^{\prime}\right) \operatorname{var}(\pi)-\left(\operatorname{cov}\left(r_{m}^{\prime}, \pi\right)\right)^{2}} \\
& \beta_{2 i}=\frac{\operatorname{cov}\left(r_{i}, \pi\right) \operatorname{var}\left(r_{m}^{\prime}\right)-\operatorname{cov}\left(r_{i}, r_{m}^{\prime}\right) \operatorname{cov}\left(r_{m}^{\prime}, \pi\right)}{\operatorname{var}\left(r_{m}^{\prime}\right) \operatorname{var}(\pi)-\left(\operatorname{cov}\left(r_{m}^{\prime}, \pi\right)\right)^{2}}
\end{aligned}
$$

É possível obter uma representação em variáveis econômicas para $a_{0}, a_{1}$ e $a_{2}$ com o auxilio de um portfólio $Z$ que tenha retorno real médio $E\left(r_{Z}\right)=a_{0}$ e que seja ortogonal aos fatores de mercado e infiação. Nestes termos obtém-se $a_{1}=E\left(r_{m}^{\prime}\right)-E\left(r_{Z}\right) \mathrm{e}$ $a_{2}=E\left(r_{Z}\right)-E\left(r_{F}\right)$. A validade destas espressões depende também do uso de uma aproximação linear para a taxa de inflação. É importante observar que esta linearidade permite, adicionalmente, uma formulação do modelo EGR em termos nominais. De fato, neste caso, se $R_{i}$ representa o retorno nominal do ativo, então $r_{i}=R_{i}-\pi$. Segue-se que

$$
E\left(R_{i}\right)=R_{F}+\left(E\left(R_{m}\right)-R_{F}\right) \beta_{1 i}+\left(E\left(R_{Z}\right)-R_{F}\right) \beta_{2 i}
$$

onde $R_{m}$ é o retorno nominal de um portfólio que contém somente os $n$ ativos de risco. 
As fórmulas para os coeficientes de sensibilidade podem também ser escritas com as variáveis nominais, com a substituição de $r_{m}^{\prime}$ por $R_{m}$ e de $r_{i}$ por $R_{i}$. Esta é a formulação mais conveniente do modelo EGR para nossa abordagem econométrica.

A derivação detalhada das fórmulas e resultados acima pode ser depreendida de Tannuri (1992).

\section{3. $O$ ajuste estatístico do modelo EGR.}

A fim de ajustar o modelo EGR, em primeira instância, estimamos os coeficientes de sensibilidade $\beta_{1 i}$ e $\beta_{2 i}$ de cada ativo. Isto foi feito a partir de séries temporais (1976-1985) para a taxa de inflação, para um índice geral de mercado e para os retornos nominais dos ativos financeiros de interesse. Consideramos em nosso estudo 58 ativos escolhidos a partir de sua representatividade medida pelo índice de liquidez reportado no Informe Técnico BOVESPA (Bolsa de Valores do Estado de São Paulo). A Tabela 1 lista estes ativos classificados em cinco grupos de atividades (indústria de transformação, indústria mecânica e de siderurgia, empresas públicas, bancos e financeiras e outras empresas), seus rendimentos médios nominais no período e os valores que encontramos para os coeficientes de sensibilidade. Nestes cálculos utiliza-se a representação dos coeficientes em termos nominais. Como proxies para os valores de $R_{m}$ utilizamos as taxas de vari ação percentual mensais do IBOVESPA (Índice da Bolsa de Valores do Estado de São Paulo). Esta escolha se justifica pelo fato de que, na realidade, esse índice representa a única peça de informação global disponível sobre a evolução dos rendimentos no mercado de nosso interesse. Maiores detalhes sobre a composição do IBOVESPA e suas limitações como índice de mercado podem ser vistas em Tannuri (1992). Como proxies para as taxas de inflação utilizamos as variaçōes percentuais mensais do IGPDI (Índice Geral de Preços, Disponibilidade Interna). Com o objetivo de eliminar correlações espúrias da variação do IGPDI com as variações do IBOVESPA, ajustamos, às vari ações do IGPDI, um processo autoregressivo de primeira ordem com uma componente sistemática de tendência. Este modelo não é rejei tado pelo teste de Dickey-Fuller e fornece uma alternativa para a modelagem de processos não estacionários como sugerido em Fuller 
(1976). A Tabela 2 apresenta os resultados deste ajuste. A coluna correspondente a BKS apresenta o valor do teste spectral de Bartlet, Smirnov e Kolmogorov. ${ }^{1}$ O teste suporta a hipótese de ruído branco para a série residual do $\triangle I G P D I$. Como medida de retorno nominal para os ativos utilizamos a série das taxas de lucratividade mensal. ${ }^{2}$

A adequabilidade do modelo EGR pode ser estudada, condicionalmente aos valores observados para os coeficientes de sensibilidade, através de uma regressão do tipo cross-section. Nesta abordagem utiliza-se o rendimento médio dos ativos no período como variável dependente. Preliminarmente ao ajuste desta regressão mostrou-se conveniente a classificação das ações em cinco grupos de atividades. O Grupo 1 engloba as ações de indústrias de transformações, o Grupo 2 engloba as ações de indústrias mecânica e de siderurgia, o Grupo 3 engloba as ações de empresas públicas, o Grupo 4 engloba as ações de bancos e financeiras privadas e o Grupo 5 engloba as demais ações. A Tabela 1, na coluna Grupo, mostra a classificação das ações que escolhemos. É importante enfatizar aqui que esta estratificação foi feita na tentativa de melhorar a representatividade do modelo EGR. Este seria, certamente, o caso, na presença de interação dos retornos esperados com os diferentes tipos de ativos. A Tabela 3 apresenta os resultados estatísticos que obtivemos. A regressão foi feita com variáveis instrumentais com a utilização da técnica sugerida por Durbin (Johnston, 1984). As variáveis $D_{2}, D_{3}, D_{4}$ e $D_{5}$ são dummies indicadoras dos respectivos grupos de ações. Os coeficientes destas variáveis medem os efeitos dos Grupos, i.e., diferenças de médias ajustadas pelos coeficientes de sensibilidade, tendo por base o Grupo 1. Vê-se que o modelo EGR é significante e que apesar do poder de previsão baixo (medido por $R^{2}$ ). Esta parece ser uma característica de tais modelos a julgar pelos resultados publicados na literatura. Veja por exemplo Roll e Ross (1980) e Chen (1983). Vale a pena mencionar aqui que os resultados podem ser melhorados se considerarmos apenas o periodo 1981-1985. Veja Tannuri (1992) para maiores detalhes a esse respeito.

${ }^{1}$ Veja Fuller (1976) e Dickey e Brocklebank (1986).

2 As taxas de lucratividade (contabilidade ajustada) mensais foram obtidas do Informe Técnico BOVESPA. 
Dos Grupos considerados somente o Grupo 2 apresenta retorno médio nominal significativamente diferente (menor) que os demais. Embora não reportado na Tabela 3, não observamos interação entre os Grupos e os coeficientes de sensibilidade. A influência do coeficiente de sensibilidade a inflação é marginal e a do coeficiente de sensibilidade ao mercado é significante $(5 \%)$ e parece dominar a relação. Em outras palavras, estes resultados sugerem que os investidores sejam mais sensiveis aos riscos de mercado do que aos riscos de inflação. Neste contexto os resultados obtidos são consistentes com a teoria no que tange aos sinais obtidos para os coeficientes de sensibilidade.

Com o objetivo de validar a estrutura em dois fatores do EGR procedemos a uma análise TAP dos retornos reais dos ativos. A análise fatorial via o método de extração dos fatores principais (ver Mardia, Bibby e Kent, 1979) sugere a presença de oito construtos ou fatores abstratos. Estes, conjuntamente, representam pouco mais de $70 \%$ da variação total observada nos retornos dos ativos. A Tabela 4 apresenta os autovalores associados a cada construto e a participação rela tiva de cada um na variabilidade total. Parece evidente que devamos concentrar nossa atenção no Construto 1, responsável por $40 \%$ da variação total. A associação deste construto com os fatores de mercado e inflação representa forte indicação empírica da adequabilidade da hipótese do modelo EGR, pelo menos no caso em que estudamos e, de certa forma, sugere as razões do poder de previsão obti do para o modelo na Tabela 2.

Para medir o grau de associação do Construto 1 com os fatores de interesse calculamos os scores desta variável no tempo e utilizamos estes valores como respostas de uma variável dependente numa regressão com a taxa de variação mensal do IGPDI e a taxa de variação mensal do IBOVESPA. Os resultados deste ajuste aparecem na Tabela 5. As indicações são fortes no sentido da associação, como facilmente se depreende das estatísticas da Tabela 5.

\section{Conclusões.}

O modelo EGR, ajustado as séries históricas (1976-1985) dos retornos nominais dos principais ativos da Bolsa de Valores de São Paulo, é significante mas tem poder de previsão baixo. A sensibili- 
dade aos riscos de mercado domina a sensibilidade aos riscos decorrentes da inflação que apresenta apenas significância marginal. Os retornos médios nominais das ações de indústrias mecânica e de siderurgia, ajustados pelos níveis de sensibilidade ao risco, são significativamente inferiores aos demais. $\mathrm{Na}$ abordagem TAP o único construto obtido com representatividade substancial na variabilidade dos retornos reais dos ativos é explicado significativamente pela taxa de variação mensal do IBOVESPA e pela taxa de variação mensal do IGPDI. Isto sugere a validade da hipótese de dois fatores do modelo de Elton, Gruber e Rentzler (1983).

(Submetido em maio de 1993. Revisado em setembro de 1993)

\section{Referências}

Chen, N. 1983. "Some empirical tests of the theory of arbitrage pricing." The Journal of Finance 5 38:1393-1414.

Dickey, A. D. \& Brocklebank, J. C. 1986. SAS System for Forecasting Time Series. North Carolina: SAS Institute.

Elton, E., Gruber M. \& Rentzler J. 1983. "The arbitrage pricing model and returns on assets under uncertain inflation." The Journal of Finance 2 38:525-537.

Fuller, W. A. 1976. Introduction to Statistical Time Series. New York: John Wiley.

Johnston, J. 1984. Econometric Methods. New York: McGrawHill, Inc.

Mardia, K. V., Bibby, J. M. \& Kent, J. T. 1979. Multivariate Analysis. New York: Academic Press.

Merton, R. 1973. "An intertemporal capital asset pricing model." Econometrica 5 41:867-887.

Roll, R. \& Ross, S. A. 1980. "The empirical investigation of the arbitrage pricing theory." The Journal of Finance 5 35: 1073-1103.

Ross, S. A. 1976. "The arbitrage theory of capital asset pricing." Journal of Economic Theory 13:341-360.

Ross, S.A. 1977. "Return, risk and arbitrage." In Friend I. \& Bicksler J., eds., Risk and Return in Finance, vol. I. Cambridge, Mass.: Ballinger. 
Tannuri, M.E.A. 1992. "O impacto da inflação sobre os retornos dos ativos: ajuste de um modelo APT para o mercado acionário de São Paulo, 1976-1985." Dissertação de mestrado, Departamento de Economia, UnB.

Tabela 1.

Açōes: Grupos, coeficientes de sensibilidade (betas)

e retornos médios mensais (valores absolutos).

\begin{tabular}{lccrr}
\hline Aç̃o & Grupo & Beta (1) & Beta (2) & Retorno \\
\hline Anderson Clayton op & 1 & 0,6942 & 1,3859 & 10,4967 \\
Brahma pp & 1 & 0,5234 & 0,2077 & 7,0958 \\
Cacique pp & 1 & 0,7558 & $-0,8352$ & 11,7275 \\
Cica pp & 1 & 0,5941 & 1,1736 & 7,1333 \\
Souza Cruz op & 1 & 0,6722 & 1,1142 & 8,8308 \\
Arno pp & 1 & 0,3583 & 0,4706 & 7,5383 \\
Consul pp & 1 & 0,3807 & 0,4738 & 11,2008 \\
Ericson op & 1 & 0,7804 & $-1,4034$ & 9,1683 \\
Pireli op & 1 & 0,7384 & 0,6518 & 8,9658 \\
Duratex pp & 1 & 0,6509 & 1,7241 & 8,9075 \\
Estrela pp & 1 & 0,6719 & 1,2623 & 8,7817 \\
Alpargatas pp & 1 & 0,6340 & $-0,0971$ & 9,2058 \\
Artex pp & 1 & 0,5926 & 0,4428 & 9,7258 \\
Hering pp & 1 & 0,5572 & 0,7422 & 9,1825 \\
Guararapes op & 1 & 0,5041 & $-0,4084$ & 9,9058 \\
Vidra. Santa Marina op & 1 & 0,6609 & 1,0192 & 10,2758 \\
Moinho Santista op & 1 & 0,6739 & $-0,2823$ & 9,5925 \\
Casa Anglo op & 1 & 0,4212 & $-0,4759$ & 9,3650 \\
Lojas Americanas op & 1 & 0,2306 & 0,5203 & 8,3567 \\
Mesbla pp & 1 & 0,8119 & 0,7706 & 8,6142 \\
\hline
\end{tabular}


Tabela 1.

Continuação.

\begin{tabular}{lccrr}
\hline Açäo & Grupo & Beta (1) & Beta (2) & Retorno \\
\hline FNV PPA & 2 & 1,1438 & $-0,6264$ & 9,1138 \\
Metal Leve pp & 2 & 0,6958 & 0,7273 & 9,2258 \\
Bardela pp & 2 & 0,7345 & $-0,7052$ & 9,5050 \\
Cobrasma pp & 2 & 1,1341 & $-1,6777$ & 8,2717 \\
Ind. Vilares pp & 2 & 1,1899 & 1,4745 & 10,0975 \\
Eluma pp & 2 & 0,9003 & $-0,8305$ & 8,4300 \\
Fundicăo Tupy pp & 2 & 0,6890 & $-0,5191$ & 8,0550 \\
Acesita op & 2 & 0,9281 & 2,3950 & 5,2400 \\
Aq̣s Vilares pp & 2 & 1,3427 & $-1,2626$ & 9,6483 \\
Belgo Mineira op & 2 & 0,8657 & 1,6513 & 8,2867 \\
Sid. Aø̣orte pp & 2 & 0,6972 & 0,8158 & 7,1958 \\
Petrobrás on & 3 & 0,9225 & 2,0204 & 8,3700 \\
Petrobrás pp & 3 & 1,0012 & 0,8553 & 8,5875 \\
Vale do Rio Doce pp & 3 & 1,0663 & 0,6994 & 8,6908 \\
Banco do Brasil on & 3 & 0,6983 & $-0,4285$ & 8,7067 \\
Banco do Brasil pp & 3 & 0,8450 & $-0,1579$ & 8,9158 \\
Banespa on & 3 & 0,7693 & $-0,8717$ & 9,7525 \\
Banespa pp & 3 & 0,9672 & $-0,5722$ & 10,6017 \\
Banco Bandeirantes pp & 4 & 0,6414 & $-2,4781$ & 7,9292 \\
Banco Itaú on & 4 & 0,6532 & $-1,1411$ & 9,3075 \\
\hline
\end{tabular}


Tannuri, Souza, Caron

Tabela 1.

Continuação.

\begin{tabular}{lcrrr}
\hline Açä & Grupo & Beta (1) & Beta (2) & Retorno \\
\hline Bradesco pn & 4 & 0,5565 & $-0,8061$ & 10,2058 \\
Banco Nacional pn & 4 & 0,3251 & $-1,0385$ & 6,3200 \\
Banco Real pn & 4 & 0,6974 & $-0,8446$ & 8,6425 \\
Unibanco on & 4 & 0,4899 & 1,5045 & 8,1792 \\
Bradesco Inv. pn & 4 & 0,5453 & $-0,0759$ & 9,7000 \\
Real Inv. pn & 4 & 0,7437 & 0,2188 & 10,1375 \\
Bradesco Fin. pn & 4 & 0,3169 & 0,5719 & 10,2267 \\
Real Fin. pn & 4 & 0,6767 & $-0,4998$ & 11,3583 \\
Itausa pn & 4 & 0,6479 & 0,1809 & 10,5208 \\
Const. Beter pp & 5 & 1,2269 & $-3,6336$ & 11,7758 \\
Mendes Junior pp & 5 & 0,8472 & $-0,3184$ & 12,1508 \\
Copas pp & 5 & 0,9999 & 5,4878 & 10,1833 \\
Telesp pe & 5 & 0,3205 & 1,4922 & 9,0750 \\
Cesp pp & 5 & 0,5331 & 3,2098 & 7,4433 \\
Paulista Forca e Luz op & 5 & 0,9121 & 2,5571 & 7,9167 \\
Cemig pp & 5 & 0,7135 & $-0,2918$ & 8,3900 \\
Varig pp & 5 & 1,0901 & $-0,9372$ & 10,8750 \\
Brasmotor op & 5 & 0,4951 & 0,4756 & 9,6575 \\
\hline
\end{tabular}

Tabela 2.

Modelo de série temporal para variaçōes do IGPDI. $\Delta I G P D I_{t}=\alpha_{0}+\alpha_{1} \Delta I G P D I_{t-1}+\alpha_{2} t+\epsilon_{t}$

\begin{tabular}{lccccc}
\hline Variável & Estimativa & Desvio & $t$ & $R^{2}$ & BKS \\
\hline Entercepto & 0,7788 & 0,3225 & 2,4149 & 0,7646 & 0,1009 \\
CagAIGPDI & 0,4661 & 0,0825 & 5,6497 & & \\
Tempo & 0,0430 & 0,0077 & 5,5844 & & \\
\hline
\end{tabular}


Ajuste de um modelo de arbitragem

Tabela 3.

Relação retorno médio-risco com variáveis indicadoras

(Dummies) por setor de atividade.

$R M_{i}=\gamma_{0}+\gamma_{1} D_{2}+\gamma_{2} D_{3}+\gamma_{3} D_{4}+\gamma_{4} D_{5}+$

$+\gamma_{5} \beta_{1 i}+\gamma_{6} \beta_{2 i}+u_{i} \quad i=1 . .58$.

\begin{tabular}{lrrrl}
\hline Variável & Estimativa & Desvio & $t$ & $R^{2}$ \\
\hline Intercepto & 8,0761 & 0,6254 & 12,9135 & 0,2542 \\
$D_{2}$ & $-1,5357$ & 0,5514 & $-2,7851$ & \\
$D_{3}$ & $-0,7945$ & 0,5986 & $-1,3273$ & \\
$D_{4}$ & $-0,0560$ & 0,4696 & $-0,1193$ & \\
$D_{5}$ & 0,2290 & 0,5280 & 0,4337 & \\
Beta 1 & 2,0848 & 0,9231 & 2,2585 & \\
Beta 2 & $-0,2686$ & 0,1320 & $-2,0348$ & \\
\hline
\end{tabular}

Tabela 4.

Análise fatorial modelo TAP.

\begin{tabular}{crr}
\hline Construto & Autovalor & Proporcão \\
\hline 1 & 16,8783 & 0,4024 \\
2 & 3,0022 & 0,0716 \\
3 & 2,2184 & 0,0529 \\
4 & 1,9715 & 0,0470 \\
5 & 1,7996 & 0,0429 \\
6 & 1,5911 & 0,0379 \\
7 & 1,4723 & 0,0351 \\
8 & 1,3308 & 0,0317 \\
\hline
\end{tabular}


Tannuri, Souza, Caron

Tabela 5.

Validade do modelo em dois fatores: regressão dos scores fatoriais nas variaçōes do IGPDI e do IBOVESPA.

\begin{tabular}{lrrrcc}
\hline Variável & Estimativa & Desvio & $t$ & $R^{2}$ & DW \\
\hline Intercepto & 0,0271 & 0,1194 & 0,2270 & 0,6396 & 2,076 \\
$\triangle$ IGPDI & $-0,0742$ & 0,0178 & $-4,1685$ & & \\
$\triangle$ IBOVESPA & 0,0546 & 0,0038 & 14,3684 & & \\
\hline
\end{tabular}


